\title{
La apropiación del espacio del otro en la literatura hispanoamericana: ¿sumisión o liberación?
}

\author{
Luz Aurora Pimentel \\ Universidad Nacional Autónoma de México (UNAM) \\ pimentelanduiza@gmail.com
}

En un primer momento, habría que pensar que la representación del espacio en el relato se cumple en buena medida por la descripción de los lugares y objetos que lo pueblan conformando así el escenario indispensable para toda acción narrada. Ahora bien, la descripción como práctica textual para dar cuenta del espacio puede darse desde la virtualidad del nombre y del nombre propio hasta la extrema expansión de la serie, el inventario o el catálogo que serían sus rasgos definitorios. Pero la serie no tiene forma, necesita algo que le ponga coto, que le dé sentido; un modelo, un esquema. La interacción entre la serie predicativa y el modelo de organización que le da forma y sentido establece el sistema descriptivo que está en la base de la representación verbal del espacio en los textos narrativos. Con frecuencia el espacio representado se va construyendo a partir del entreverado narración-descripción a cargo de un narrador más o menos impersonal, o bien filtrado por la conciencia reflectora del personaje focal. En todo caso, ese espacio representado tiene una primerísima e ineludible función de basamento, de escenario de la acción humana. No obstante, ni aun como escenario es el espacio representado algo neutro o inocente, debido al modelo mismo de organización que lo pone en contacto con la realidad extratextual.

Los modelos de organización espacial, especialmente en los textos realistas, tienden a coincidir con los esquemas de saber y de poder de una época y/o de una cultura dada. Es por ello que las representaciones del espacio pasan por esquemas intelectuales que, por abstractos, los hace reconocibles. Pero habría que matizar la naturaleza de estos esquemas de saber y de poder que organizan la representación del espacio. El espacio representado es, como dice Henri Lefebvre, "el espacio concebido. . . es el espacio dominante en una sociedad. . . Las representaciones del espacio estarían penetradas de saber (una mezcla de conocimiento e ideología) siempre relativo y en transformación" $(48,51)$. Ahora bien, ese espacio representado se relativiza y transforma no solo en el tiempo sino en su interacción con los actores y con el contexto, la época y la cultura en los que ha sido representado. En ese sentido, el espacio representado deviene espacio de representación en el momento en que los personajes o el propio narrador confrontan su estatus ideológico o de poder 


\section{La apropiación del espacio del otro en la literatura hispanoamericana...}

Luz Aurora Pimentel

para dotarlo de nuevas atribuciones, nuevas significaciones y/o nuevas funciones. Porque justamente, acudiendo nuevamente a Lefebvre, "El espacio de representación [es] el espacio vivido a través de las imágenes y símbolos que lo acompañan, y por ende espacio de 'habitantes', de 'usuarios', pero también de algunos artistas. . . es el espacio dominado, y por tanto experimentado, del que la imaginación se intenta apropiar y modificar" (49), y es por ello que "un espacio apropiado semeja una obra de arte" (192).

En una buena parte de la narrativa hispanoamericana es evidente esta confrontación. Baste pensar en algunos cuentos de Cortázar, como "Carta a una señorita en París", "Fin de etapa", "La noche boca arriba" o "Axóltl”, en los que se confrontan dos espacios de poder, dos espacios culturales antagónicos. Evidentemente, la relación de los personajes con el espacio representado no es solamente de apropiación y de transformación, sino puede ser también de sujeción o de imposición, como en el caso de "Fin de etapa". O bien el espacio impuesto por la cultura dominante del otro es asumido como el único posible y repetido en representaciones intertextuales sin fin, como ocurre en las representaciones de la naturaleza americana en el siglo XIX a partir del modelo del Paul et Virginie [Pablo y Virginia], de Bernardin de Saint-Pierre, a fines del siglo XVIII, y cuyo ejemplo más acabado e influyente fue la María, de Jorge Isaacs —ambos descendientes lejanos, finalmente, del antiquísimo topos del locus amoenus. ${ }^{1}$

Quisiera abordar ahora algunos textos de Carpentier y de Donoso para examinar más de cerca esta interesante dinámica de la representación. Comenzaré con la descripción de un almacén en el segundo capítulo de El siglo de las luces, de Alejo Carpentier. Tras la muerte del padre, Carlos tendrá que encargarse del negocio; pero ahora, con su hermana Sofía y su primo Esteban, recorre el almacén; un bien heredado, una obligación impuesta, un espacio racional, ordenado, pero esencialmente carcelario para los adolescentes. No obstante, la descripción, en su composición misma, habla ya de una rebelión:

. . abrieron la puerta que conducía a la casa aledaña, donde se tenía el comercio y el almacén, ahora cerrado por tres días a causa del duelo. Tras de los escritorios y cajas fuertes, empezaban las calles abiertas entre montañas de sacos, toneles, fardos de todas procedencias. Al cabo de la Calle de la Harina, olorosa a tahonas de ultramar, venía la Calle de los Vinos de Fuencarral, Valdepeñas y Puente de la Reina, cuyas barricas goteaban el tinto por todas las canillas, despidiendo alientos de bodega. La Calle de los Cordajes y Jar-

1 Para un análisis más detallado de los modos de representación de la naturaleza en la narrativa del siglo XXI ver mi artículo, "The Representation of Nature in Nineteenth-Century Narrative and Iconography” (2004 156-172). 
cias conducía al hediondo rincón de pescado curado, cuyas pencas sudaban la salmuera sobre el piso. Regresando por la Calle de los Cueros de Venado, los adolescentes volvieron al Barrio de las Especias, con sus gavetas que pregonaban, de solo olerlas, el jengibre, el laurel, los azafranes y la pimienta de la Veracruz. Los quesos manchegos se alineaban sobre tablados paralelos, conduciendo al Patio de los Vinagres y Aceites en cuyo fondo, bajo bóvedas, se guardaban mercancías disparatadas: hatos de barajas, estuches de barbería, racimos de candados, quitasoles verdes y rojos, molinillos de cacao, con las mantas andinas traídas de Maracaibo, el desparramo de los palos de tintura y los libros de hojas para dorar y platear, que venían de México. (21)

Es este, sin duda, un espacio de abundancia y de orden; el espacio del poder económico que extiende sus redes allende el mar. El modelo taxonómico espacial que organiza la descripción acomoda cuidadosamente los bienes por especies: los quesos alineados, los vinos goteando en el derroche de su riqueza; hasta lo misceláneo tiene su lugar con un toque de lo exótico — las mantas andinas de Maracaibo, la pimienta de la Veracruz. Todo rezumante, todo oloroso; todo se paladea, comenzando por las palabras. Este es, claramente, el espacio representado del poder colonial, ostensiblemente económico, aunque por sus redes ultramarinas, evidentemente político: un espacio de poder impuesto que reclama la participación de Carlos. Sin embargo, los personajes ingresan en este espacio no para someterse a él sino para transformarlo, de un espacio potencialmente claustrofóbico y abrumador, en un espacio lúdico que los libera. El modelo cultural suplementario que tan bellamente lo organiza — el de la arquitectura urbana- le da esa otra dimensión al almacén, lo deja, por decirlo así, a cielo abierto. Deja de ser un espacio para el comercio y para el incremento del poder económico, para convertirse en un lugar donde se puede pasear y jugar. En efecto, los adolescentes no hacen el inventario de sus riquezas, se pasean por sus calles, avenidas, plazas y barrios, deleitando sus sentidos todos, del mismo modo que, más tarde, el inventario de sus riquezas, embaladas en un "laberinto de cajas", se convertirá en un paisaje "alpestre", lleno de "puentes", "cumbres" y "riscosos vericuetos":

De día en día se había ido edificando un laberinto de cajas dentro de la casa, donde cada cual tenía su rincón, su piso, su nivel, para aislarse o reunirse en conversación. . Había como una rampa, un camino alpestre, que salía del quicio del salón, pasando por sobre un armario recostado, para subir a las Tres Cajas de Vajilla, puestas una sobre otra, desde las cuales podía contemplarse el paisaje de abajo, antes de ascender por riscosos vericuetos de tablas rotas. . . hasta la Gran Terraza. . . En los caminos y mesetas, escondrijos y puentes, se daba cada cual a leer lo que le pareciera. (25-26) 


\section{La apropiación del espacio del otro en la literatura hispanoamericana...}

Luz Aurora Pimentel

Como concluye el narrador, "todo era transfigurado por un juego perpetuo que establecía nuevas distancias con el mundo exterior” (28). Es así como, por el milagro de la metáfora, un espacio impuesto se convierte en un espacio transfigurado, un espacio dinámico, vivido en la imaginación e incluso en la cotidianidad, modificando sus usos y funciones. Habría que hacer notar que, en esta transformación del espacio representado en espacio de representación, un factor central además del modelo de organización suplementario, es la mediación del cuerpo: es el movimiento, la actitud corporal misma de pasear, en lugar de inventariar, el elemento transformador del espacio.

Si bien la transformación de este espacio representado en espacio vivido es el resultado de los actos de estos adolescentes soñadores, es el discurso narrativo-descriptivo del narrador el que da cuenta, por medio de sus descripciones metafóricas, de esta apropiación del espacio representado para convertirlo en un espacio de representación vivo.

Ahora bien, puede suceder que sea el propio narrador, sin la concurrencia de los actos de los personajes, quien subvierta la representación del espacio del otro, exhibiéndola como lo que es: una imposición. Esto ocurre en otra novela de Carpentier, El reino de este mundo, en la magistral descripción del Palacio de Sans-Souci del Rey Henri Christophe:

[Ti Noel] se había detenido, maravillado por el espectáculo más inesperado, más imponente que hubiera visto en su larga existencia. Sobre un fondo de montañas estriadas de violado por gargantas profundas se alzaba un palacio rosado, un alcázar de ventanas arqueadas, hecho casi aéreo por el alto zócalo de una escalinata de piedra. A un lado había largos cobertizos tejados, que debían de ser las dependencias, los cuarteles y las caballerizas. Al otro lado, un edificio redondo, coronado por una cúpula asentada en blancas columnas, del que salían varios sacerdotes de sobrepelliz. A medida que se iba acercando, Ti Noel descubría terrazas, estatuas, arcadas, jardines, pérgolas, arroyos artificiales y laberintos de boj. Al pie de pilastras macizas, que sostenían un gran sol de madera negra, montaban la guardia dos leones de bronce. Por la explanada de honor iban y venían, en gran tráfago, militares vestidos de blanco, jóvenes capitanes de bicornio, todos constelados de reflejos, sonándose el sable sobre los muslos. Una ventana abierta descubría el trabajo de una orquesta de baile en pleno ensayo. A las ventanas del palacio asomábanse damas coronadas de plumas, con el abundante pecho alzado por el talle demasiado alto de los vestidos a la moda. En un patio, dos cocheros de librea daban esponja a una carroza enorme, totalmente dorada, cubierta de soles en relieve. Al pasar frente al edificio circular del que habían salido los sacerdotes, Ti Noel vio 
que se trataba de una iglesia, llena de cortinas, estandartes y baldaquines, que albergaba una alta imagen de la Inmaculada Concepción.

Pero lo que más asombraba a Ti Noel era el descubrimiento de que ese mundo prodigioso, como no lo habían conocido los gobernadores franceses del Cabo, era un mundo de negros. Porque negras eran aquellas hermosas señoras, de firme nalgatorio, que ahora bailaban la rueda en torno a una fuente de tritones; negros aquellos dos ministros de medias blancas, que descendían, con la cartera de becerro debajo del brazo, la escalinata de honor; negro aquel cocinero, con cola de armiño en el bonete, que recibía un venado de hombros de varios aldeanos conducidos por el Montero Mayor; negros aquellos húsares que trotaban en el picadero; negro aquel Gran Copero, de cadena de plata al cuello, que contemplaba, en compañía del Gran Maestre de Cetrería, los ensayos de actores negros en un teatro de verdura; negros aquellos lacayos de peluca blanca, cuyos botones dorados eran contados por un mayordomo de verde chaqueta; negra, en fin, y bien negra, era la Inmaculada Concepción que se erguía sobre el altar mayor de la capilla, sonriendo dulcemente a los músicos negros que ensayaban una salve. Ti Noel comprendió que se hallaba en Sans-Souci, la residencia predilecta del rey Henri Christophe, aquel que fuera antaño cocinero en la calle de los Españoles, dueño del albergue de La Corona, y que hoy fundía monedas con sus iniciales, sobre la orgullosa divisa de Dios, mi causa y mi espada. (88-90)

Lo que más llama la atención en este pasaje es que se trata de una descripción cuasi musical, en dos tempos, por así decirlo, una suerte de andante en blanco mayor, y un presto en negro mayor. Fuera del tono rosado del palacio y las montañas estriadas de violado, en la primera parte de la descripción todo es blanco o connota la blancura: "blancas columnas", "militares vestidos de blanco", "estatuas", "plumas", "lacayos de peluca blanca", la "Inmaculada Concepción". De hecho, habría que matizar: más que connotar la blancura, en términos puramente cromáticos, las alusiones son a lo europeo y de manera específica, a Versalles. Más aún, podríamos afirmar que subyace en esta descripción una configuración cultural —el Palacio de Versalles - responsable de la organización de todos los elementos de la descripción. Es notable que la serie predicativa no se ordene en torno a ningún modelo de organización espacial ostensible: “terrazas, estatuas, arcadas, jardines, pérgolas, arroyos artificiales y laberintos de boj" forman una serie aparentemente sin orden ni concierto; sin embargo, hay un orden espacial, incluso secuencial, subyacente: el plano de Versalles.

El Palacio de Versalles, en sí mismo diseñado tanto como representación del mundo como representación del poder de Luis XIV sobre el mundo, está emplaza- 


\section{La apropiación del espacio del otro en la literatura hispanoamericana...}

Luz Aurora Pimentel

do en un promontorio que permite abarcar con la mirada, es decir dominar, los tres órdenes de la naturaleza: el primero, la naturaleza domeñada por el hombre para el deleite de sus sentidos — los jardines, con sus estatuas, pérgolas, laghettos, fuentes y laberintos de boj- ; el segundo, la naturaleza cultivada, sustentadora de vida las huertas, viñedos y cultivos - ; y, finalmente, la naturaleza supuestamente "salvaje", desde luego artificial también, creada para ser "espectáculo" y "escenario" de cacería para el rey. Este espacio doblemente representado — mundo y poder sobre el mundo- sirvió luego para la construcción de muchos otros palacios europeos, Schöenbrunn, Nymphenburg, Aranjuez... infinitos espejos y espejismos del poder mundano.

Es este espacio, triplemente representado ahora en el Caribe, el que hace que, en una primera lectura, se active tal configuración cultural y veamos en Sans-Souci otro Versalles, sin siquiera nombrarlo. Pero ahí están las palabras, casi como objetos, como entidades autónomas; términos cultural y temporalmente marcados que remiten a objetos igualmente marcados y que, como lo veremos más tarde, develan un fenómeno tanto cultural como semántico: el objeto, y la palabra que lo designa, hace mundo. En este caso, la sola constelación de objetos-palabras remite a una configuración descriptivo-cultural implícita que organiza toda la descripción, le da un sentido y construye una imagen, un mundo: Versalles, completo hasta con la carroza que alude a este nuevo Rey Sol caribeño. Es por ello que la configuración versallesca, como imán, jala a los actores hacia lo europeo y los colorea de blanco. Y si hemos hablado de esta primera parte de la descripción como una suerte de andante en blanco mayor, podemos ahora precisar que se trata de un blanco más bien racial que cromático, debido a la imantación de la configuración cultural. De ahí la enorme sorpresa y el deleite de la segunda parte de la descripción. En apariencia es una repetición; se trata de lo mismo: los mismos actores, es cierto, pero en negro, con el remate rítmico y juguetón, "negra, en fin, y bien negra, era la Inmaculada Concepción".

Ahora bien, es evidente que, aunque Ti Noel es el personaje focal de esta descripción, ya que se nos propone como la deixis de referencia espacial móvil, cuyos ojos van registrando todo lo ahí descrito, su mirada es, de hecho, una pantalla neutra sobre la que la otra, irónica y subversiva, la mirada del narrador, va exhibiendo el ridículo de semejante boato afrancesado. No es pues el punto de vista ni cognitivo ni ideológico de Ti Noel el que anima esta descripción, sino la perspectiva del narrador; una perspectiva no solo irónica sino ambivalente, pues, al mismo tiempo que denuncia la sumisión al espacio y a los esquemas culturales del otro, por el solo ritmo lúdico de la descripción, transforma esta sumisión en una apropiación creadora y, por ende, liberadora: sí, "negra. . . y bien negra, era la Inmaculada Concepción”. 
Un ejemplo más de la imposición del espacio del otro que lleva a una apropiación y luego a una transformación en espacio de representación lo constituye Casa de campo, de José Donoso. José Donoso, ese gran destructor de espacios. Es cierto que ningún espacio ficcional es estático, si tan solo por efecto de las diversas modalidades del tiempo del relato que lo van modificando, porque el devenir y las diversas acciones van modificando los espacios representados, de tal suerte que, por la presión misma de la temporalidad del relato, el espacio mismo se temporaliza. Podríamos hablar entonces de la transformación del espacio en términos de una temporalización del espacio, como veremos más tarde.

Más aún, en estas diversas formas de temporalización del espacio se van dando, de manera compleja, las transformaciones que permiten el paso de un espacio representado a un espacio de representación, de un espacio-escenario a un espacio apropiado, destruido-reconstruido, deformado o transfigurado, pero siempre resignificado en su interacción con los personajes. Pero nadie tan destructor y transgresor de espacios como Donoso. Baste recordar el deplorable estado en el que queda el impecable piso prestado en El jardín de al lado, o la casa infinitamente tapiada hasta convertirla en una "casa imbunche", proyección de la identidad del Mudito en El obsceno pájaro de la noche.

En Casa de campo tenemos otro Versalles, pero oligárquico esta vez. Mismo modelo, sin embargo; mismo procedimiento descriptivo, curiosamente. Como Versalles, la casa está "posada sobre un levantamiento del terreno" (75):

Su parque de castaños, tilos y olmos, sus amplios céspedes por donde deambulaban los pavos reales, la diminuta isla de rocaille en el laghetto de aguas ahogadas por papiros y nenúfares, el laberinto de boj, el rosedal, el teatro de verdura poblado de personajes bergamascos, las escalinatas, las ninfas de mármol, las ánforas, remedaban solo los modelos más exaltados, desterrando toda nota que lo comprometiera con lo autóctono. El parque, enclavado en esa llanura sin un solo árbol que manchara su extensión, era como una esmeralda, su profundidad cuajada de fantásticos jardines de materia más dura que la materia del paisaje. (57)

Nótese, nuevamente, el recurso a la serie sin ningún otro modelo de espacialidad que organice la descripción; no obstante, la configuración descriptivo-cultural subyacente nos indica en qué dirección va la perspectiva. A diferencia de la de Carpentier que se mueve de la terraza hacia los jardines, la de Donoso va de la lejanía de la "isla de rocaille en el laghetto" a "las escalinatas", las "ninfas de mármol" y "las ánforas". La deixis de referencia espacial parece detenerse en la terraza. Este narrador, émulo de los decimonónicos, asume la perspectiva de los dueños de la casa, aceptando este espacio de poder impuesto desde el extranjero como un 'modelo 


\section{La apropiación del espacio del otro en la literatura hispanoamericana...}

Luz Aurora Pimentel

exaltado' que 'destierra toda nota' que comprometa el espacio representado 'con lo autóctono’. El poder de clase de los Ventura y la sujeción de los nativos están simbólicamente representados, en primera instancia, por la voz decimonónica que los identifica y con los que se identifica y, en segunda, por esta casa de campo versallesca, espacio de poder extranjero ocioso y parasitario, como las inútiles, nocivas gramíneas igualmente importadas en un tiempo remoto ya olvidado. Ahora bien, este poder impuesto está representado no solo por el modelo versallesco de los jardines que rodean a la casa sino por una configuración descriptivo-cultural mucho más compleja:

La casa, posada sobre un levantamiento del terreno apenas más perceptible que un suspiro en el cuerpo tendido de la llanura, se hallaba construida encima de un intrincado panal de bóvedas y galerías ahuecadas en innumerables niveles de profundidad. . En los aledaños nacía el laberinto de pasadizos, de los cuales brotaban alvéolos, cuevas, celdillas, aberturas, cámaras abatidas por repentinas sábanas de telarañas y frecuentados por animalitos mucilaginosos e inofensivos que casi no se movían. . También en las inmediaciones se hallaba el cultivo de hongos, pálidos, gordos como el vientre de un sapo, cuyo encargado, al poco tiempo de encierro en el subterráneo, llegaba a ser tan frío, tan quieto, tan ciego como esas deliciosas criptogramas a que los señores eran tan aficionados. Bastaba avanzar con un candil en la mano. . p para llegar a otros sistemas de celdillas del panal. Poco tardaban en darse cuenta de que si avanzaran hacia el lado de donde venía el frío por los túneles, entre líquenes y filtraciones, abriéndose paso por abandonados jardines de hongos que producían aberrantes especies barrocas como el cáncer, hacia cavernas y pasillos construidos de antigua piedra o socavados en materia natural donde chisporroteaban cristales, podían encontrar cosas desazonantes. Pero ninguno de los Ventura bajaba jamás al subterráneo. (75-76)

El modelo espacial dominante en la descripción de la casa de los Ventura está centrado en las categorías arriba y abajo que organizan y orientan el movimiento descriptivo.2 Es significativo que, en esta visión de conjunto, el trabajo textual se concentre en los subterráneos. La metáfora sostenida del panal, como otro sistema de organización descriptiva superpuesto al espacial, le da un alto grado de cohesión y de iconización a los subterráneos. Desde el punto de vista ideológico hay aquí un claro privilegio del espacio de abajo, connotado positivamente como labor productiva gracias a la metáfora del panal. El "arriba" por no estar sujeto a un desarrollo —-más allá del que parentéticamente define a la casa como un

2 Para un análisis más detallado del espacio en esta novela, ver mi ensayo "La dimensión ideológica del espacio en Casa de campo" en El espacio en la ficción (165-185). 
suspiro- queda propuesto solo como una cubierta, de suyo insignificante, apenas un suspiro, que oculta los tesoros y el potencial productivo de este ignorado panal. Yuxtapuesto a este espacio productivo está el "aberrante", el de los "hongos, pálidos, gordos como el vientre de un sapo", las "deliciosas criptogramas a que los señores eran tan aficionados".

A lo largo de toda la novela, hay indicaciones fragmentarias del valor y significación reales de estos subterráneos. Pero no es sino ya muy avanzado el relato que el narrador nos ofrece un "panorama histórico", transformando así la significación de las otras descripciones:

Al edificar una casa encima de la mina [de sal] y defender el predio reservado al solaz de la familia por medio del cerco de lanzas, los Ventura encerraron todos los accesos a la mina, reservándose, sin embargo, los más importantes como entradas a aquella parte de los sótanos, donde establecieron algunas dependencias de la casa. Terminó así, en una generación, rápidamente, el trabajo de la sal y se olvidó su importancia, instaurándose en cambio la explotación del oro en delgadas láminas. . . Fue así como la sal dejó de representar la autonomía de los nativos, y, por lo tanto, el peligro. Los Ventura, entonces, pudieron sellar definitivamente la mina con un voluntarioso olvido de los pólipos de túneles y cavernas sobre los que la mansión se alzaba, que no tardaron en quedar reducidos en sus memorias a la conocida topografía de la parte utilizada, y a un incierto "un poco más allá", también de fácil manejo. Algo después, cuando ya se olvidó del todo el motivo del emplazamiento de la casa. . . los Ventura, como para hacer gala de su ignorancia, no dejaban de preguntarse. . qué diablos podía haber impulsado al tatarabuelo a emplazar allí su casa (350).

El maravilloso paraje subterráneo cobra ahora una dimensión ideológica capital. El panal, como campo semántico que organiza metafóricamente esta significación ideológica, multiplica sus eslabones: la miel en tanto que asociada al panal es un oro líquido que nutre y sustenta la vida, como el comercio de sal otrora sustentara la vida física y social de los nativos. El correlato negativo del oro líquido en el panal subterráneo es el oro laminado que por su rigidez mineral no sirve para nutrirlos sino para minar su salud: para los nativos el oro solo significa miseria e insalubridad. Es así como las minas subterráneas, metaforizadas en panal, significan un pasado productivo; en cambio, las minas arriba, en las montañas "azules", solo sirven para mantener el statu quo de la ficción "azul" de los Ventura y de la explotación de los nativos.

La oposición puramente espacial arriba/abajo cobra ahora una significación ideológica gracias a la metáfora del panal; pero, sobre todo, gracias a la equivalencia espaciotemporal arriba $=$ presente, abajo $=$ pasado: arriba la dominación 


\section{La apropiación del espacio del otro en la literatura hispanoamericana...}

Luz Aurora Pimentel

de los Ventura de reciente importación, abajo el pasado de los nativos reprimido violentamente. Más aún, debido a la peculiar configuración descriptiva de estas dos entidades, la casa de campo construida encima de las minas de sal entra en relación metafórica con fenómenos arquitectónicos muy parecidos que solo se dan en el contexto histórico de la colonización: la casa de campo construida sobre las minas es un equivalente metafórico de un fenómeno típico de la conquista: la catedral, iglesia o capilla construida sobre la pirámide o templo ceremonial prehispánico. Ilustra, además y de manera muy concreta, la realización histórica de una misma configuración con valor descriptivo ideológico, de un mismo espacio de poder impuesto representado por esta configuración descriptivo-cultural.

Así, el espacio sellado vale como la figuración no solo del poder de los colonizadores sino del olvido: forma privilegiada de la abolición del tiempo y de la destrucción de una tradición. Como en el caso de las gramíneas, los Ventura, responsables iniciales de esta alteración del espacio natural y del cultural, en su infinita pereza e inutilidad acaban por olvidar que son ellos los responsables de la alteración, ellos los actuales "propietarios" de ignorados tesoros. El olvido y la ignorancia se nos presentan, así, como la forma más brutal de aniquilación de una civilización, del divorcio cultural y temporal entre la cultura dominante y la autóctona.

Es interesante subrayar el papel multifacético que juega el narrador en la representación del espacio en esta novela. Por una parte, lo hemos visto, es él quien asumiendo la perspectiva de poder del espacio representado impuesto nos ofrece una primera descripción de la casa de los Ventura como un espacio durable. Irónicamente, conforme avanza la novela, esa perspectiva irá cambiando, junto con la propia voz del narrador. Pasajes, como este último, son discursos más bien de corte historiográfico que nos alejan considerablemente de esa voz inicial y nos hablan del espacio soterrado que tiene todo el potencial de volver a convertirse en un espacio de representación para los nativos. De tal suerte que el discurso narrativo-descriptivo anuncia o acompaña la transformación del espacio representado en Casa de campo por las acciones de los personajes. Tomemos solamente un par de ejemplos. La reja que circunda el parque: una cerca que deviene cerco, con varillas a los que los nativos restituyen su función y sentido original: lanzas. Nuevamente, el paso del espacio representado al espacio de la representación se opera por el milagro de la metáfora. En un primer momento, el objeto se designa como "varilla" que remata en "punta"; el material de que está hecha la varilla es hierro, la punta es de "metal amarillo". Pero de manera insidiosa comienzan a infiltrarse descripciones metafóricas de la misma reja que acaban por suplantar a las del plano denotativo: las varillas se convierten en "lanzas", el metal amarillo en "oro". En un primer momento, la designación "lanzas/oro" queda inscrita en el contexto de la reja, el cual orienta la interpretación de "lanzas" y "oro" exclusivamente como metáforas: 
las varillas, mientras solo conforman la cerca, parecen lanzas; el metal amarillo, oro. Pero poco a poco los contextos comienzan a variar: de protección la reja deviene prisión. Se van anulando así las equivalencias y oposiciones que tan cómodamente se habían establecido antes del viaje de los Ventura. En ausencia de los adultos, "adentro" ya no equivale a la protección sino al encierro; como se han llevado todo medio de transporte, se pone de manifiesto la naturaleza carcelaria del exterior que entonces ya no significa libertad sino prisión. Si las equivalencias y oposiciones simétricas se anulan; si ambos términos, dentro y fuera, significan prisión, la oposición espacial básica interior/exterior comienza a resquebrajarse, aguardando su total desmoronamiento.

Es precisamente el vaivén entre lo metafórico y lo literal lo que permitirá a Donoso, más tarde, transformar el espacio diegético construido y, con él, la identidad y función de los objetos que lo pueblan. En el caso de la reja, las lanzas se nos revelarán no como una designación metafórica sino como verdaderas y "literales" lanzas, como ese signo de poder de los nativos que, en otro tiempo, fuera "desemantizado" por la dominación de los Ventura. Encerradas en una reja que les niega su identidad como lanzas, signos desvirtuados y obligados a significar en un contexto ajeno, la empalizada de hierro ha protegido a los Ventura, volviéndose contra los nativos, anulándolos al excluirlos. Dada esta oposición irreconciliable, la libertad de las lanzas no puede sino significar la de los nativos. Solo liberándose del espacio de poder impuesto por los Ventura serán capaces los nativos de actuar en la libertad de un espacio de representación que les permita restituir funciones, símbolos y significados a los objetos que pueblan su mundo, aun cuando en la feroz lucha de poderes y de espacios destruidos y reconstruidos de esta novela este espacio de representación de los nativos dure apenas un suspiro también.

Un último ejemplo de esta subversión del espacio representado es el espacio "restaurado" por los sirvientes. Lo interesante en este caso es que el espacio de representación construido por los sirvientes no es más que una copia servil, grotesca y deformada del espacio de poder representado, lo cual exhibe el servilismo de los Ventura con respecto a la cultura europea, revelándose así estos "señores" como lo que en realidad son: no amos sino sirvientes. En ausencia de los amos, los otros sirvientes, los oficiales, se encargan de "restaurar" el trompe l'oeil, fresco que "engaña a la mirada" proponiendo perspectivas y puertas falsas que acaban por contaminar de irrealidad, o por lo menos de ambigüedad, a las puertas y perspectivas reales. Pero en vez de restaurarlo, los sirvientes lo desfiguran hasta convertirlo en algo grotesco, en una proyección pictórica de esta nueva casta dominante, aunque también transitoria:

Cuanto su pincel de restaurador tocaba parecía transformarse en un engendro alucinado. Sus esbirros, suspendidos por andamios y poleas a distintas alturas 


\section{La apropiación del espacio del otro en la literatura hispanoamericana...}

Luz Aurora Pimentel

sobre la faz del fresco, se encargaban de transformar imperceptiblemente a las diosas retozonas en arpías, las nubes sonrosadas en tormentas (323).

En el silencio del salón de baile —un oído tísico hubiera podido percibir la inefable caricia de los pinceles sobre la piel del cuello de un adolescente para mancharlo de pústulas (325).

La desfiguración del trompe l'oeil es, a un tiempo, la venganza de los sirvientes y el emblema de su asunción al poder. Pero esta nueva forma del poder no es más que una réplica degradada y desfigurada del reinado de los Ventura; su emblema es el trompe l'oeil, al que los sirvientes han convertido en un verdadero "engaño", o un engaño verdadero, tal vez. Fresco convertido en un monstruo que acaba por ingresar en el mundo de lo supuestamente real. A partir del reinado de los sirvientes hasta el final, el trompe l'oeil entabla un diálogo de reflejos con lo animado hasta cancelar toda demarcación posible entre ficción y realidad.

Casa de campo es emblemática de la representación simbólica de las luchas de poder no solo entre los diversos miembros de una familia, entre las distintas clases, razas y culturas, sino entre los distintos tiempos de la historia de una comunidad; luchas de poder simbolizadas en las incesantes — casi podríamos decir, alucinantes - transformaciones del espacio diegético. Es, sin duda una novela que ilustra hasta qué punto el espacio representado en la ficción va mucho más allá de fungir simplemente como el marco o escenario de las acciones de los personajes. Porque los personajes interactúan con el espacio, ya sea de manera pasiva o activa, modificándolo, obligándolo a pasar de ser un espacio representado, abstracto, esquemático, a ser un espacio vivido, un espacio de representación al que se le pueda transformar, modificar, incluso deformar; al que se le puedan atribuir las significaciones más diversas, subjetivas o colectivas, contribuyendo, de este modo, a perfilar las dimensiones temáticas y simbólicas del relato. 
CARTOGRAFíAS E IMAGINARIOS DE LA LITERATURA CHILENA: ESPACIOS, TEMPORALIDADES Y SUJETOS

\section{Obras citadas}

Carpentier, Alejo. El siglo de las luces. Barcelona: Seix Barral, 1965.

_.. El reino de este mundo. Barcelona: Seix Barral, 1983.

Donoso, José. Casa de campo. Barcelona: Seix Barral, 1978 y 1983.

Lefebvre, Henri. La production de l'espace. Paris: Anthropos, 2000.

Pimentel, Luz Aurora. "The Representation of Nature in Nineteenth-Century Narrative and Iconography". Literary Cultures of Latin America. A Comparative History, Vol. II. Eds. Mario J. Eds. Valdés y Djelal Kadir. Inglaterra: Oxford University Press, 2004. 156-172.

- "Modos de representación del espacio en la narrativa de ficción" "La multiplicidad del sujeto: construcción y disolución de la identidad narrativa". Constelaciones I. Ensayos de teoría narrativa y Literatura Comparada. México: UNAM / Iberoamericana Verveurt / Bonilla, 2012.

- "La multiplicidad del sujeto: construcción y disolución de la identidad narrativa". Constelaciones I. Ensayos de teoría narrativa y Literatura Comparada. México: UNAM / Iberoamericana Verveurt / Bonilla, 2012.

- El espacio en la ficción/ficciones espaciales: la representación del espacio en los textos narrativos. México: Siglo XXI / UNAM, 2001. 